\title{
Mildly explosive autoregression under stationary conditional heteroskedasticity*
}

\author{
Stelios Arvanitis \\ Athens University of Economics and Business \\ Tassos Magdalinos \\ University of Southampton
}

18 January 2018

\begin{abstract}
A limit theory is developed for mildly explosive autoregressions under stationary (weakly or strongly dependent) conditionally heteroskedastic errors. The conditional variance process is allowed to be stationary, integrable and mixingale, thus encompassing general classes of GARCH type or stochastic volatility models. No mixing conditions nor moments of higher order than 2 are assumed for the innovation process. As in Magdalinos (2012), we find that the asymptotic behaviour of the sample moments is affected by the memory of the innovation process both in the form of the limiting distribution and, in the case of long range dependence, the rate of convergence, while conditional heteroskedasticity affects only the asymptotic variance. These effects are cancelled out in least squares regression theory and thereby the Cauchy limit theory of Phillips and Magdalinos (2007a) remains invariant to a wide class of stationary conditionally heteroskedastic innovations processes.
\end{abstract}

Keywords: Central limit theory, Explosive autoregression, Long Memory, Conditional heteroskedasticity, GARCH, mixingale, Cauchy distribution.

${ }^{*}$ Magdalinos acknowledges research support by the British Academy under grant SG151171. Arvanitis acknowledges research support by the Research Centre of AUEB, EP-2215-01/00-01. 


\section{Introduction}

First order autoregressive processes with an explosive root, i.i.d. Gaussian innovations and zero initial condition were first analysed by White (1958), who using a moment generating function technique, derived a Cauchy limit theory for the OLS/ML estimator. Using martingale methods, Anderson (1959) arrived to the same conclusion and showed that the Cauchy limit theory is not invariant to deviations from Gaussianity and that, in general, the limit distribution of the OLS/ML estimator depends on the distribution of the (i.i.d.) innovations.

Invariance of the Cauchy least squares regression limit theory to the distribution of the innovations can be recovered when the explosive root approaches unity as the sample size $n$ tends to infinity at sufficiently slow rate. Phillips and Magdalinos (2007a, hereafter $\mathrm{PM}_{a}$ ) considered mildly explosive processes of the form

$$
X_{t}=\rho_{n} X_{t-1}+u_{t}, \quad \rho_{n}=1+\frac{c}{n^{\alpha}}, \alpha \in(0,1), c>0 .
$$

When the innovation process $\left(u_{t}\right)_{t \in \mathbb{Z}}$ is i.i.d. and square integrable, $\mathrm{PM}_{a}$ establish central limit theorems for sample moments generated by mildly explosive processes and obtain the following least squares regression theory:

$$
\frac{1}{2 c} n^{\alpha} \rho_{n}^{n}\left(\hat{\rho}_{n}-\rho_{n}\right) \Rightarrow \mathcal{C} \quad \text { as } n \rightarrow \infty
$$

This Cauchy limit theory is invariant to both the distribution of the innovations and to the initialization of the mildly explosive process.

The results of $\mathrm{PM}_{a}$ were generalised by Phillips and Magdalinos (2007b, hereafter $\mathrm{PM}_{b}$ ) to include a class of weakly dependent innovations. Aue and Horvath (2007) relaxed the moment conditions on the innovations by considering an i.i.d. innovation sequence that belongs to the domain of attraction of a stable law. The limiting distribution is represented by a ratio of two independent and identically distributed stable random variables and reduces to a Cauchy distribution when the innovations have finite variance. Multivariate extensions are included in Magdalinos and Phillips (2008).

Magdalinos $\left(2012\right.$, hereafter $\mathrm{M}_{a}$ ) considered mildly explosive autoregressions generated by a correlated innovation sequence that may exhibit long range dependence. The asymptotic behaviour of the sample moments that

appear in the ratio of the centred least squares estimator $\hat{\rho}_{n}-\rho_{n}$ was found 
to be affected by long range dependence both in the rate of convergence and in the form of the limiting distribution, crucially, in the same way and by the same amount for both components of the ratio. Hence, there is an asymptotic cancellation and, unlike its constituent components, the ratio $\hat{\rho}_{n}-\rho_{n}$ is not affected by the memory of the innovation sequence and continues to be asymptotically Cauchy with the rate of convergence of (2). The limit theory of $\mathrm{PM}_{a}$ was thus generalised and found invariant to the dependence structure of the innovation sequence even in the long memory case.

Phillips, Wu and Yu (2009) and Phillips and Yu (2011) employ the limit theory of $\mathrm{PM}_{a}$ to construct inferential procedures for the detection and dating of financial bubbles. Since the empirical stylized facts of financial asset returns are consistent with conditional heteroskedasticity, see for example Ghysels et al. (1996), it is natural to ask whether these Cauchy based confidence intervals remain valid in the presence of time varying conditional second moments. Lee (2017) and Oh, Lee and Chan (2017) confirm the Cauchy limit theory of $\mathrm{PM}_{a}$ for conditionally heteroskedastic innovations under restrictive assumptions on the innovation sequence $u_{t}$ that include strong mixing with exponentially decaying coefficients, the existence of fourth moments and, in the case of Lee (2017), restrictions on the distribution of the conditional variance in some neighborhood of the origin. In it well known that finite four moments impose severe restrictions on the parameter space of GARCH type models. Also, the fact that the Cauchy limit theory (2) is directly generalisable to long memory innovations that violate the strong mixing condition, suggests that strong mixing may not be an appropriate medium of testing the invariance of the Cauchy limit theory (2) to the dependence and distributional properties of $u_{t}$.

In this paper we extend the homoskedastic framework of $\mathrm{M}_{a}$ and that of Lee (2017) and Oh et.al. (2017) by allowing the sequence $u_{t}$ in (1) to be a stationary (possibly long memory) linear process with to be constructed upon a stationary square integrable conditionally heteroskedastic process where the conditional variance is a mixingale. The innovation sequence $u_{t}$ is not assumed to be strong mixing nor to have finite moments of higher order than 2. We provide detailed examples of general classes of conditionally heteroskedastic models that satisfy our framework, including stationary $\mathrm{ARCH}(\infty)$ processes, asymmetric GARCH type models and loglinear stochastic volatility models in Examples 1-3 in the next section. Our asymptotic development is based on the establishment of a new law of large numbers for weakly dependent heterogeneous triangular arrays (Lemma 1) 
below, which constitutes a partial generalisation of the $L_{1}$-mixingale law of large numbers in Andrews (1988). Employing this law of large numbers and following the martingale approximation approach of $\mathrm{M}_{a}$, we establish the invariance of the Cauchy limit theory (2) under this extended dependence and conditional heteroskedasticity framework. In doing so, we confirm the robustness of the Phillips, Wu and Yu (2009) and Phillips and Yu (2011) procedures in environments consistent with the empirical properties of financial asset returns.

\section{Main Results}

Consider the mildly explosive process in (1) with innovations $\left(u_{t}\right)_{t \in \mathbb{N}}$ that take the form of a covariance stationary linear process with possible long memory, as in $\mathrm{M}_{a}$ (see Assumption LP below). We propose a framework for the introduction of conditional heteroskedasticity to the innovations of (1) that: (i) maintains the potential for strong dependence in the innovation sequence (by avoiding to impose mixing conditions on $\left(u_{t}\right)_{t \in \mathbb{N}}$ ); (ii) does not require the existence of moments of higher order than 2 for $\left(u_{t}\right)_{t \in \mathbb{N}}$, thus giving rise to GARCH-type models with sufficiently general parameter spaces. We impose this framework on the primitive innovations $\left(\varepsilon_{t}\right)_{t \in \mathbb{Z}}$ of the linear process $u_{t}$ in (1) by Assumption $\mathrm{CH}$ bellow. We denote conditional expectation by $\mathbb{E}_{\mathcal{F}}(\cdot)$ and the $L_{p}$ norm by $\|\cdot\|_{p}$.

Assumption CH. The process $\left(\varepsilon_{t}\right)_{t \in \mathbb{Z}}$ satisfies $\varepsilon_{t}=z_{t} \sqrt{h_{t}}$ a.s. for all $t \in \mathbb{Z}$, where the sequence $\left(z_{t}\right)_{t \in \mathbb{Z}}$ is i.i.d. with $\mathbb{E}\left(z_{1}\right)=0$ and $\mathbb{E}\left(z_{1}^{2}\right)=1$. Given a filtration $\left(\mathcal{F}_{t}\right)_{t \in \mathbb{Z}}, h_{t}$ is $\mathcal{F}_{t-1}$-adapted and $z_{t}$ is independent of $\mathcal{F}_{t-1}$ for all $t \in \mathbb{Z}$. The process $\left(z_{t}, h_{t}\right)_{t \in \mathbb{Z}}$ is strictly stationary with $h_{t}>0$ a.s. and $\sigma^{2}:=\mathbb{E}\left(h_{1}\right) \in(0, \infty)$. Finally, there exist real positive sequences $\left(\zeta_{t}\right)_{t \in \mathbb{Z}}$ and $\left(\psi_{m}\right)_{m \in \mathbb{N}}$ satisfying $\sup _{t \in \mathbb{Z}} \zeta_{t}<\infty, \psi_{m} \rightarrow 0$ as $m \rightarrow \infty$, and

$$
\left\|\mathbb{E}_{\mathcal{F}_{t-1-m}}\left(h_{t}-\sigma^{2}\right)\right\|_{1} \leq \zeta_{t} \psi_{m} \text { for any } t, m \geq 0 .
$$

Under Assumption $\mathrm{CH},\left(\varepsilon_{t}\right)_{t \in \mathbb{Z}}$ is conditionally heteroskedastic w.r.t. $\left(\mathcal{F}_{t}\right)_{t \in \mathbb{Z}}$. Typically, $\mathcal{F}_{t}$ represents the informational content of the history, up to time $t$, of the i.i.d. process $\left(z_{t}\right)_{t \in \mathbb{Z}}$ or any other process upon which $h_{t}$ is formed. Furthermore, $\left(\varepsilon_{t}\right)_{t \in \mathbb{Z}}$ is a stationary white noise with variance equal to $\sigma^{2}$, and additionally a martingale difference when $z_{t}$ is $\mathcal{F}_{t}$-adapted. The conditional 
variance process $\left(h_{t}\right)_{t \in \mathbb{Z}}$ is strictly stationary and integrable. The adaptation property of $h_{t}$ to $\mathcal{F}_{t-1}$ combined with (3) implies that its demeaned version $h_{t}-\sigma^{2}$ is an $L_{1}$ mixingale (see for example Andrews (1988)).

Assumption $\mathrm{CH}$ encompasses several classes of conditionally heteroskedastic processes. For example, for (strong) GARCH-type models we have that $\mathcal{F}_{t}:=\sigma\left(z_{t-i}, i \geq 0\right)$. Then, stationarity typically follows by a representation of $h_{t}$ as a measurable function of $\mathcal{F}_{t-1}$. Positivity and uniform integrability are usually ensured via properties of the aforementioned representation possibly combined with parameter restrictions. The mixingale property is readily verifiable for a large class of frequently used conditionally heteroskedastic models, such as $\mathrm{ARCH}(\infty)$ processes which include finite order covariance stationary GARCH processes; see Example 1 below. In more complicated cases, such as Examples 2 and 3 below, the mixingale property can be established by stricter integrability conditions on the primitive innovations $z_{t}$ of $h_{t}$ (only the first moment of $h_{t}$ is assumed to exist) along with strong mixing properties due to the relation between the mixingale and the strong mixing properties implied by relevant mixing inequalities, see for example McLeish (1975). Notice, however, that these sufficient conditions are not necessary: the conditional variance process $h_{t}$ of the $\operatorname{ARCH}(\infty)$ model of Example 1 satisfies the mixingale property without higher order moments nor mixing conditions. Note also that the strong mixing property of $h_{t}$ does not impose weak dependence on the innovation sequence $u_{t}$ in (1): the latter may have long memory (see Assumption LP(ii) below). In what follows, we provide details on certain general classes of models that satisfy Assumption CH.

Example $1(\operatorname{ARCH}(\infty)$ process). For $\omega>0$, some non negative real sequence $\left(\alpha_{i}\right)_{i \in \mathbb{N}}$ and $\mathcal{F}_{t}:=\sigma\left(z_{t-i}, i \geq 0\right)$ consider the infinite order recursion defining the $\operatorname{ARCH}(\infty)$ model:

$$
h_{t}=\omega+\sum_{i=1}^{\infty} \alpha_{i} z_{t-i}^{2} h_{t-i}=\omega+\sum_{i=1}^{\infty} \alpha_{i} \varepsilon_{t-i}^{2} .
$$

A sufficient condition for the existence of a unique stationary causal solution to the above is that $\sum_{i=1}^{\infty} \alpha_{i}<1$ in which case the latter admits a Volterra expansion and $\sigma^{2}=\omega\left(1-\sum_{i=1}^{\infty} \alpha_{i}\right)^{-1}$ (see Theorem 2.1 of Giraitis et al. (2000)). Furthermore, by Theorem 4.1 of Giraitis et al. (2000), for $\left(\tilde{\alpha}_{i}\right)_{i \in \mathbb{N}}$ defined by $\tilde{\alpha}(z)=\sum_{i=0}^{\infty} \tilde{\alpha}_{i} z^{i}=1 / \alpha(z)$ with $\alpha(z)=\sum_{i=0}^{\infty} \alpha_{i} z^{i}$ and $|z| \leq 1$, 
we have that

$$
\varepsilon_{t}^{2}-\sigma^{2}=\sum_{i=0}^{\infty} \tilde{\alpha}_{i} v_{t-i}
$$

with $\left(\left(v_{t}\right)_{t \in \mathbb{Z}},\left(\mathcal{G}_{t}\right)_{t \in \mathbb{Z}}\right)$ a stationary martingale difference defined by $v_{t}:=$ $\left(z_{t}^{2}-1\right) h_{t}$, for all $t \in \mathbb{Z}$. Using the above, we have that for any $t$ and any $m>0$,

$$
\left\|\mathbb{E}_{\mathcal{F}_{t-1-m}}\left(h_{t}-\sigma^{2}\right)\right\|_{1}=\left\|\mathbb{E}_{\mathcal{F}_{t-1-m}}\left(\varepsilon_{t}^{2}-\sigma^{2}\right)\right\|_{1} \leq 2 \sigma^{2} \sum_{i=m}^{\infty}\left|\tilde{\alpha}_{i}\right|,
$$

hence $(3)$ holds with $\zeta_{t}=2 \sigma^{2}$ and $\psi_{m}=\sum_{i=m}^{\infty}\left|\tilde{\alpha}_{i}\right|$, since $\sum_{i=0}^{\infty}\left|\tilde{\alpha}_{i}\right|<\infty$. Hence, the previous assertions hold also for any $\operatorname{GARCH}(p, q)$ model under the $\sum_{i=1}^{\infty} \alpha_{i}<1$ restriction when applied to its $\mathrm{ARCH}(\infty)$ representation.

Example 2 (Asymmetric GARCH type Models). Similarly to Carrasco and Chen (2002) consider the stochastic recursion

$$
\Lambda\left(h_{t}\right)=c\left(m_{t}\right) \Lambda\left(h_{t-1}\right)+g\left(m_{t}\right),
$$

with $\Lambda$ increasing and continuous on $\mathbb{R}_{+}, m_{t}$ a measurable function of $z_{t}$, and $c, g$ polynomials. This formulation encompasses several $\operatorname{GARCH}(1,1)-$ type models as for example the $(1,1)$ versions of the LGARCH, VGARCH, EGARCH, MGARCH, GJR, and TGARCH models (for definitions and references see Carrasco and Chen (2002)). Their properties are, among others and in some varying extend, in accordance with the empirical stylized fact of dynamic asymmetry in financial time series (for the so-called asymmetric leverage effects, see for example Bollerslev et al. (2011)). Suppose now that the distribution of $m_{t}$ is absolutely continuous w.r.t. the Lebesgue measure on $\mathbb{R}$ with support that contains zero, and that for some $s \geq 1$, $|c(0)|<1, \mathbb{E}\left(c^{s}\left(m_{1}\right)\right)<1$, and $\mathbb{E}\left(g^{s}\left(m_{1}\right)\right)<1$. Then, by Proposition 5 of Carrasco and Chen (2002) the adaptation and stationarity parts of Assumption $\mathrm{CH}$ follow, and furthermore the conditional variance process $h_{t}$ is strongly mixing with exponentially decaying mixing coefficients. If furthermore $\mathbb{E}\left(\left(\Lambda^{-1}\left(c\left(m_{t}\right) \Lambda\left(h_{t-1}\right)+g\left(m_{t}\right)\right)\right)^{s}\right)<+\infty$, for some $s>1$, then $\sigma^{2}=\mathbb{E}\left(h_{1}\right)$ exists and by the mixing inequality of Lemma 2 of McLeish (1975) (3) holds.

In a similar manner consider the Power $\operatorname{GARCH}(\mathrm{p}, \mathrm{q})$ recursion for $\omega>0$, 


$$
\begin{aligned}
\alpha_{i} \geq 0, i=1, \ldots, p, \beta_{i} & \geq 0, i=1, \ldots, q, \delta>0, \\
h_{t}^{\delta} & =\omega+\sum_{i=1}^{p} \alpha_{i} h_{t-1}^{\delta} z_{t-i}^{2 \delta}+\sum_{i=1}^{p} \beta_{i} h_{t-1}^{\delta},
\end{aligned}
$$

which is essentially a Box-Cox transformation for the conditional variance. If the distribution of $z_{t}$ is absolutely continuous w.r.t. the Lebesgue measure on $\mathbb{R}$ with support equal to the real line, for some $s>\frac{1}{\delta}, \mathbb{E}\left(z_{1}^{2 s \delta}\right)<\infty$, and $\mathbb{E}\left(\lambda_{\max }^{s}\left(z_{1}\right)\right)<1$ where $\lambda_{\max }$ is the largest root of $\sum_{i=1}^{\max (p, q)}\left(\alpha_{i} z_{1}^{2 \delta}+\beta_{i}\right) / \lambda^{i}$ (where a parameter is considered equal to zero if its index exceeds the relevant order), then all the assertions of Assumption $\mathrm{CH}$ follow by Proposition 13 of Carrasco and Chen (2002) and Lemma 2 of McLeish (1975).

Example 3 (Log-Linear Stochastic Volatility). Suppose now that $\left(\eta_{t}\right)_{t \in \mathbb{Z}}$ is another i.i.d. sequence such that $\left(\eta_{t}, z_{t}\right)_{t \in \mathbb{Z}}$ is stationary, $\mathcal{F}_{t}:=\sigma\left(u_{t-i}, z_{t-i}, i \geq 0\right)$, $z_{t}$ is independent of $\mathcal{F}_{t-1}$, and $\omega \in \mathbb{R}$ while $\left(\beta_{i}\right)_{i \in \mathbb{N}}$ is a real sequence. Consider the process

$$
\ln h_{t}=\omega+\sum_{i=0}^{\infty} \beta_{i} \eta_{t-i-1} .
$$

The conditional variance is defined as an (exogenous) log-linear process w.r.t. $\left(\eta_{t}\right)_{t \in \mathbb{Z}}$, and thereby the previous specify a stochastic volatility model (see for example Straumann (2004)). The possibility of contemporaneous dependence between $\eta_{t}$ and $z_{t}$ is also related to the empirical dynamic asymmetry in financial data (see above). It is easy to see that if the distribution of $\eta_{1}$ has a well-defined moment generating function, say $M_{\eta}$, on the range of $\left(\beta_{i}\right)_{i \in \mathbb{N}}$ and $\sum_{i=0}^{\infty} \ln M_{\eta}\left(\beta_{i}\right)$ converges, then all the assertions of Assumption $\mathrm{CH}$ except for (3) hold with $\sigma^{2}=\exp \left(\omega+\sum_{i=0}^{\infty} \ln M_{\eta}\left(\beta_{i}\right)\right)$. For example when $\eta_{1} \sim N(0,1)$ then square summability for the $\left(\beta_{i}\right)_{i \in \mathbb{N}}$ suffices for the above and $\sigma^{2}=\exp \left(\omega+\frac{1}{2} \sum_{i=0}^{\infty} \beta_{i}^{2}\right)$. For (3) notice that any set of conditions for strong mixing of linear processes like (5), see for example Theorem 14.9 of Davidson (1994), along with the convergence of $\sum_{i=0}^{\infty} \ln M_{\eta}\left((1+\varepsilon) \beta_{i}\right)$ for some $\varepsilon>0$ would suffice due to Theorem 14.1 of Davidson (1994), and Lemma 2 of McLeish (1975). In the standard normal case those are reduced to the absolute summability of $\left(\beta_{i}\right)_{i \in \mathbb{N}}$ due to Theorem 13.3.3. in Ibragimov and Linnik (1971).

The mixingale property of Assumption $\mathrm{CH}$ facilitates the validity of a law of large numbers for weakly dependent heterogeneous triangular arrays, 
Lemma 1(ii) below, which constitutes a partial generalisation of the $L_{1}$ mixingale law of large numbers in Andrews (1988). This is a key result for the asymptotic development of the paper, as it characterises the asymptotic behaviour of the conditional variance of martingale transforms that arise in mildly explosive least squares theory and allows the application of a central limit theorem to these martingale transforms to establish their asymptotic normality (see Lemma 2). Part (i) of the lemma is an auxiliary result of sums of martingale differences weighted by triangular arrays of constants, leading to the main result of part (ii), where the martingale difference is generalised to a $L_{1}$ mixingale processes without the imposition of rates to the original mixingale numbers. We denote by $\left(\mathcal{G}_{t}\right)_{t \in \mathbb{Z}}$ a generic filtration that need not coincide with that of Assumption $\mathrm{CH}$.

Lemma 1. For an integer valued sequence $\left(k_{n}\right)_{n \in \mathbb{N}}$ with $k_{n} \rightarrow \infty$, consider an array of real numbers $\left\{\alpha_{n, t}: 1 \leq t \leq k_{n}\right\}$ satisfying

$$
\sup _{n \in \mathbb{N}} \sum_{t=1}^{k_{n}}\left|a_{n, t}\right|<\infty \text { and } \sum_{t=1}^{k_{n}} a_{n, t}^{2} \rightarrow 0 \text { as } n \rightarrow \infty \text {. }
$$

(i) If $\left(\left(\epsilon_{t}\right)_{t \in \mathbb{Z}},\left(\mathcal{G}_{t}\right)_{t \in \mathbb{Z}}\right)$ is a uniformly integrable martingale difference process, then $\left\|\sum_{t=1}^{k_{n}} a_{n, t} \epsilon_{t}\right\|_{1} \rightarrow 0$.

(ii) Let $\left(\left(y_{t}\right)_{t \in \mathbb{Z}},\left(\mathcal{G}_{t}\right)_{t \in \mathbb{Z}}\right)$ be a uniformly integrable adapted process with zero-mean satisfying

$$
\left\|\mathbb{E}_{\mathcal{G}_{t-m}}\left(y_{t}\right)\right\|_{1} \leq \zeta_{t} \psi_{m} \text { for each } t, m \geq 0
$$

for real positive sequences $\left(\zeta_{t}\right)_{t \in \mathbb{Z}}$ and $\left(\psi_{m}\right)_{m \in \mathbb{N}}$ with $\sup _{t \in \mathbb{Z}} \zeta_{t}<\infty$ and $\psi_{m} \rightarrow 0$ as $m \rightarrow \infty$. Then $\left\|\sum_{t=1}^{k_{n}} a_{n, t} y_{t}\right\|_{1} \rightarrow 0$.

The adaptation property along with (7) imply that $\left(y_{t}\right)_{t \in \mathbb{Z}}$ is an $L_{1}$ mixingale (see for example Andrews (1988)).

Having introduced a convenient conditional heteroskedasticity framework, we proceed to defining the linear relationship between the innovations of the mildly explosive autoregression (1) with the process $\left(\varepsilon_{t}\right)_{t \in \mathbb{Z}}$ of Assumption $\mathrm{CH}$. 
Assumption LP. For each $t \in \mathbb{N}$, $u_{t}$ has Wold representation $u_{t}=\sum_{j=0}^{\infty} c_{j} \varepsilon_{t-j}$ where $\left(\varepsilon_{t}\right)_{t \in \mathbb{Z}}$ is as in Assumption $C H$ and $\left(c_{j}\right)_{j \geq 0}$ is a sequence of constants satisfying $c_{0}=1$ and one of the following conditions:

(i) $\sum_{j=0}^{\infty}\left|c_{j}\right|<\infty$ and $\sum_{j=0}^{\infty} c_{j} \neq 0$.

(ii) For each $j \in N, c_{j}=L(j) j^{-\kappa}$, for some $\kappa \in(1 / 2,1)$, where $L$ is a slowly varying function at infinity such that $\varphi(t):=L(t) t^{-\kappa}$ is eventually non-increasing and $\sup _{t \in[0, B]} t^{\delta} L(t)<\infty$ for any $\delta, B>0$.

(iii) $c_{j}=\theta j^{-1}, j \in N$, for some $\theta \neq 0$.

Assumption IC. $\quad X_{0}$ can be any fixed constant or a random process $X_{0}(n)$ satisfying $X_{0}(n)=o_{p}\left(n^{\alpha / 2}\right)$ under $L P(i), X_{0}(n)=o_{p}\left(n^{(3 / 2-\kappa) \alpha} L\left(n^{\alpha}\right)\right)$ under $L P($ ii $)$ and $X_{0}(n)=o_{p}\left(n^{\alpha / 2} \log n\right)$ under $L P($ iii $)$.

Assumptions LP and IC are identical to the assumption framework in $\mathrm{M}_{a}$. Under the first, $\left(u_{t}\right)_{t \in \mathbb{N}}$ is a covariance and strictly stationary linear process, since $\left(c_{j}\right)_{j \geq 0}$ is square summable and $\left(\varepsilon_{t}\right)_{t \in \mathbb{Z}}$ is a stationary conditionally heteroskedastic white noise.

LP(i) ensures absolute summability of the autocovariance function of $\left(u_{t}\right)_{t \in \mathbb{Z}}$ thereby giving rise to a weakly dependent stationary process. LP(ii) induces long memory to $\left(u_{t}\right)_{t \in \mathbb{Z}}$. Recall that a function $L$ is slowly varying at infinity if and only if $L(u t) / L(t) \rightarrow 1$ for any $u>0$; see Bingham, Goldie and Teugels (1987), abbreviated hereafter as BGT. The parametrisation $c_{j}=L(j) j^{-\kappa}$ is standard for stationary linear processes that exhibit long memory, see e.g. Giraitis, Koul and Surgailis (1996) and Wu and Min (2005), including stationary AFRIMA processes, for $\kappa=1-d, d \in(0,1 / 2)$ in the relevant notation. The boundary $\kappa=1$ between weak and strong dependence in the memory of the innovation sequence is investigated via the harmonic coefficients of Assumption LP(iii).

The property of $\varphi(t)$ being eventually non-increasing facilitates the computation of asymptotic variances by means of Euler summation in $\mathrm{M}_{a}$. This property is for instance satisfied by the Zygmund class of differentiable slowly varying functions (see BGT, Theorem 1.5.5). Boundedness of $t^{\delta} L(t)$ in a neighbourhood of the origin is a standard requirement for the validity of Abelian theorems for integrals involving regularly varying functions, see BGT, Proposition 4.1.2(a). Both conditions hold trivially for the stationary AFRIMA processes with fractional parameter as above, see Samorodnitsky (2006). 
Under Assumptions LP-IC, and conditional homoskedasticity for $\left(\varepsilon_{t}\right)_{t \in \mathbb{Z}}$, $\mathrm{M}_{a}$ establishes the invariance of the Cauchy regression theory of $\mathrm{PM}_{a}$. This invariance holds despite the different rates of convergence and limit distributions (that arise as a result of the memory properties of $u_{t}$ ) satisfied by the sample moments that enter least squares regression, as those are asymptotically cancelled out between the components of the OLS estimator. The question here is whether an analogous result holds under conditional heteroskedasticity. Once the appropriate asymptotic framework has been set by Assumption $\mathrm{CH}$ and the mixingale law of large numbers of Lemma 1, it turns out that not only the above invariance remains true, but also that every intermediate result of $\mathrm{M}_{a}$ continues to hold.

Specifically, as in the previous analyses of (mildly) explosive autoregression by Anderson (1959), $\mathrm{PM}_{a}$ and $\mathrm{M}_{a}$, the limit theory of the OLSE for $\rho_{n}$ depends on properties of the stochastic sequences

$$
Y_{n}(\kappa)=\frac{1}{n^{\left(\frac{3}{2}-\kappa\right) \alpha}} \sum_{t=1}^{\tau_{n}(\beta)} \rho_{n}^{-t} u_{n+1-t} \quad \text { and } \quad Z_{n}(\kappa)=\frac{1}{n^{\left(\frac{3}{2}-\kappa\right) \alpha}} \sum_{t=1}^{\tau_{n}(\beta)} \rho_{n}^{-t} u_{t}
$$

with $\rho_{n}=1+c / n^{\alpha}$ as defined in (1) and

$$
\tau_{n}(\beta)=\left\lfloor\frac{n^{\beta}}{2}\right\rfloor \quad \text { for some } \beta \in\left(\alpha, \min \left\{\frac{3 \alpha}{2}, 1\right\}\right) .
$$

For notational convenience, following $\mathrm{M}_{a}$, we employ the notation $Y_{n}(1)$ and $Z_{n}(1)$ for the sequences in (8) under both Assumptions LP(i) and LP(iii). This is consistent with the $n^{\alpha / 2}$ normalisation that applies under weak dependence.

By covariance stationarity of $\left(u_{t}\right)_{t \in \mathbb{Z}}, Y_{n}(\kappa)$ and $Z_{n}(\kappa)$ have equal variance; their asymptotic variance is computed in Lemma 1 of $\mathrm{M}_{a}$ for any white noise process $\left(\varepsilon_{t}\right)_{t \in \mathbb{Z}}$ with variance equal to $\sigma^{2}$ and is given by

$$
V_{\kappa}:=\sigma^{2} c^{2 \kappa-3} \frac{\Gamma(1-\kappa)^{2}}{2 \cos \{\pi(1-\kappa)\}}, \quad \kappa \in(1 / 2,1)
$$

where $\Gamma(x)=\int_{0}^{\infty} u^{x-1} e^{-u} d u$ is the gamma function. Under Assumption CH, the above expression for $V_{\kappa}$ continues to apply with $\sigma^{2}=\mathbb{E}\left(h_{1}\right)$. Lemma 2 below provides a limit theory $Y_{n}(\kappa)$ and $Z_{n}(\kappa)$ under conditional heteroskedasticity and both short and long memory in the innovations. Both the rates of convergence and the limit distributions depend crucially on the 
linear dependence properties of $\left(u_{t}\right)_{t \in \mathbb{Z}}$ via the memory parameter $\kappa$. Under $\mathrm{CH}$ the conditional variance process $\left(h_{t}\right)_{t \in \mathbb{Z}}$ does not affect the convergence rates, but affects the limit distributions via $\sigma^{2}=\mathbb{E}\left(h_{1}\right)$. For this value of $\sigma^{2}$, denote $\omega^{2}:=\sigma^{2}\left(\sum_{j=0}^{\infty} c_{j}\right)^{2}$.

Lemma 2. Under Assumptions $C H$ and $L P$, the sequences $Z_{n}(\kappa)$ and $Y_{n}(\kappa)$ in (8) have the following joint asymptotic behaviour as $n \rightarrow \infty$ :

(i) Under $L P(i),\left[Y_{n}(1), Z_{n}(1)\right] \Rightarrow\left[Y_{1}, Z_{1}\right]$, where $Y_{1}$ and $Z_{1}$ are independent $N\left(0, \omega^{2} / 2 c\right)$ random variables.

(ii) Under $L P(i i), L\left(n^{\alpha}\right)^{-1}\left[Y_{n}(\kappa), Z_{n}(\kappa)\right] \Rightarrow\left[Y_{\kappa}, Z_{\kappa}\right]$, where where $Y_{\kappa}$ and $Z_{\kappa}$ are independent $N\left(0, V_{\kappa}\right)$ random variables and $V_{\kappa}$ is given by (10).

(iii) Under $L P\left(\right.$ iii), $\left(\log n^{\alpha}\right)^{-1}\left[Y_{n}(1), Z_{n}(1)\right] \Rightarrow\left[Y_{1}^{\prime}, Z_{1}^{\prime}\right]$, where $Y_{1}^{\prime}$ and $Z_{1}^{\prime}$ are independent $N\left(0, \sigma^{2} \theta^{2} / 2 c\right)$ random variables.

Lemma 2 shows that Lemmata 2-4 of $\mathrm{M}_{a}$ continue to hold under conditional heteroskedasticity with $\sigma^{2}$ arising as the expectation of the conditional variance process $h_{t}$ of Assumption $\mathrm{CH}$ (instead of the conditional homoskedasticity assumption $h_{t}=\sigma^{2}$ for all $t$ a.s. maintained in $\mathrm{M}_{a}$ ). The key insight is the application of the mixingale law of large numbers of Lemma 1(ii) which ensures the validity of a standard martingale central limit theorem in each of the cases (i)-(iii) above. The joint asymptotic behaviour of $Y_{n}(\kappa)$ and $Z_{n}(\kappa)$ completely determines the limit theory of the sample moments of $X_{t}$ and of the normalised and centred OLS estimator in the context of (1), as long as the standard approximation argument of Anderson (1959) continues to apply under Assumptions CH, LP, IC. The validity of this approximation argument is established in the following lemma.

Lemma 3. Let $L$ denote an arbitrary slowly varying function at infinity. Then, under Assumptions CH, LP and IC,

$$
\begin{aligned}
\frac{\rho_{n}^{-2 n}}{n^{\alpha} n^{(3-2 \kappa) \alpha} L\left(n^{\alpha}\right)^{2}} \sum_{t=1}^{n} X_{t-1}^{2} & =\frac{1}{2 c}\left[\frac{1}{L\left(n^{\alpha}\right)} Z_{n}(\kappa)\right]^{2}+o_{p}(1) \\
\frac{\rho_{n}^{-n}}{n^{(3-2 \kappa) \alpha} L\left(n^{\alpha}\right)^{2}} \sum_{t=1}^{n} X_{t-1} u_{t} & =\frac{Y_{n}(\kappa)}{L\left(n^{\alpha}\right)} \frac{Z_{n}(\kappa)}{L\left(n^{\alpha}\right)}+o_{p}(1)
\end{aligned}
$$


as $n \rightarrow \infty$ where: (a) Under $L P(i), \kappa=1$ and $L(x)=1$. (b) Under LP(ii), $\kappa \in(1 / 2,1)$ and $L$ satisfies $L P($ ii). (c) Under LP(iii), $\kappa=1$ and $L(x)=\log x$.

Lemma 3 simply asserts that Lemma 5 of $\mathrm{M}_{a}$ continues to apply when the conditional homoskedasticity assumption of $\mathrm{M}_{a}$ is replaced by Assumption $\mathrm{CH}$ and confirms the validity of the standard approximation argument pertaining to (mildly) explosive sample moments. Combing Lemmata 2 and 3, we deduce that, under appropriate normalisation, joint convergence in distribution of $\left(\sum_{t=1}^{n} X_{t-1} u_{t}, \sum_{t=1}^{n} X_{t-1}^{2}\right)$ applies in all cases LP(i)-LP(iii). Moreover, the same normalisation applies to the centred OLS estimator $\hat{\rho}_{n}-\rho_{n}$ irrespective of the dependence properties of $u_{t}$. The resulting Cauchy limit distribution for the normalised and centred OLS estimator is a simple corollary of the continuous mapping theorem and the fact that the limiting random vectors $\left(Y_{1}, Z_{1}\right),\left(Y_{\kappa}, Z_{\kappa}\right)$ and $\left(Y_{1}^{\prime}, Z_{1}^{\prime}\right)$ of Lemma 2 consist of independent components.

Theorem 1. For the mildly explosive process generated by (1) under Assumptions $C H, L P$ and IC, the following limit theory applies as $n \rightarrow \infty$ :

$$
\frac{1}{2 c} n^{\alpha} \rho_{n}^{n}\left(\hat{\rho}_{n}-\rho_{n}\right) \Rightarrow \mathcal{C} \quad \text { as } n \rightarrow \infty,
$$

where $\mathcal{C}$ denotes a standard Cauchy random variable.

\section{Remarks.}

1. Theorem 1 shows that standard Cauchy mildly explosive regression theory continues to hold under stationarity, weak or strong linear dependence, mixingale conditional variance and second order integrability for the innovation process. Even in this general framework, the limit theory depends only on the parameters $c$ and $\alpha$ that determine the degree of mild explosion, i.e. the neighbourhood of unity that contains the mildly explosive root $\rho_{n}$. As remarked in $\mathrm{M}_{a}$ and also holds true in the current conditional heteroskedasticity context, this invariance of least squares limit theory to the memory properties of the innovation sequence is due to the strength of the (mildly) explosive regression signal. Exponential signal strength gives rise to a fundamental property of explosive and mildly explosive autoregression, established in our context by Lemma 3, that the asymptotic behaviour of the normalised and 
centred least squares estimator is completely characterised by the ratio $Y_{n}(\kappa) / Z_{n}(\kappa)$ in which the numerator and the denominator have identical rates and limiting distributions (by Lemma 2). Hence any idiosyncratic characteristic of the limit theory of the individual components $Y_{n}(\kappa)$ and $Z_{n}(\kappa)$ is essentially canceled out in the ratio. Apart from strict stationarity of $u_{t}$ (which is inherent in GARCH-type processes), Theorem 1 constitutes a generalisation of the corresponding theorem of $\mathrm{M}_{a}$.

2. Mildly explosive autoregression with conditionally heteroskedastic innovations has recently been investigated by Lee (2017) and Oh et al. (2017) under a more restrictive framework. In particular, the innovation sequence $\left(u_{t}\right)_{t \in \mathbb{Z}}$ is assumed to be strong mixing with exponentially decaying coefficients and finite fourth moments (equivalently finite second moments for the conditional variance process $h_{t}$ ). It is well known that higher order moment assumptions severely restrict the parameter space of GARCH type models. We avoid this problem since Assumption $\mathrm{CH}$ does not require the existence of second moments (or inverse second moments) for the conditional variance process. Also, Assumption $\mathrm{CH}$ does not require the innovation sequence $\left(u_{t}\right)_{t \in \mathbb{Z}}$ to be strong mixing. Example 1 shows that Assumption $\mathrm{CH}$ is satisfied by $\mathrm{ARCH}(\infty)$ processes (and hence all $\operatorname{GARCH}(p, q)$ processes) satisfying the standard stability condition, irrespective of whether $h_{t}$ is strong mixing. Even when the mixingale condition (3) is verified by the strong mixing property of $h_{t}$ (Examples 2 and 3 ), $\left(u_{t}\right)_{t \in \mathbb{Z}}$ will not be strong mixing under the strongly dependent correlation schemes of Assumption LP(ii) and LP(iii). To our knowledge, Assumption $\mathrm{CH}$ provides the most general framework of conditional heteroskedasticity in the literature of (mildly) explosive autoregressions. Further generalisation may be possible, in the direction of non-integrability of the conditional variance process, with the truncated first moment of $h_{1}$ being slowly varying at infinity, see Goldie (1991); this slow variation is likely to appear in the rates in Lemma 2, yet the Cauchy limit theory of Theorem 1 should remain unaffected. We leave such considerations for further research.

3. Theorem 1 provides a limit distribution that can be used for interval estimation. Phillips, Wu and Yu (2009) and Phillips and Yu (2011) apply the construction of Cauchy confidence intervals for the detection 
of financial bubbles. Given the fact that financial asset returns in relevant frequencies exhibit stylized facts consistent with several patterns of conditional heteroskedasticity, see for example Ghysels et al. (1996), Theorem 1 above ensures the robustness of those procedures to general form of conditional heteroskedasticity in the innovations.

\section{Proofs}

This section contains the proofs of mathematical statements in the paper. We employ a similar approach to $\mathrm{M}_{a}$ and Magdalinos (2009, hereafter $\mathrm{M}_{b}$ ) with the important addition of the mixingale law of large numbers of Lemma 1 permits the use of the martingale CLT in Corollary 3.1 of Hall and Heyde (1980) in the present framework of conditional heteroskedasticity.

\subsection{Proof of Lemma 1.}

Define $\hat{\epsilon}_{n, t}:=\epsilon_{t} \mathbf{1}\left\{\left|\epsilon_{t}\right| \leq \Delta_{n}\right\}$ and $\tilde{\epsilon}_{n, t}:=\epsilon_{t} \mathbf{1}\left\{\left|\epsilon_{t}\right|>\Delta_{n}\right\}$ for a sequence $\left(\Delta_{n}\right)_{n \in \mathbb{N}}$ satisfying

$$
\Delta_{n} \rightarrow \infty \text { and } \Delta_{n}^{2} \sum_{t=1}^{k_{n}} a_{n, t}^{2} \rightarrow 0 \text { as } n \rightarrow \infty .
$$

The martingale difference property implies that $\epsilon_{t}=\hat{\epsilon}_{n, t}-\mathbb{E}_{\mathcal{G}_{t-1}}\left(\hat{\epsilon}_{n, t}\right)+\tilde{\epsilon}_{n, t}-$ $\mathbb{E}_{\mathcal{G}_{t-1}}\left(\tilde{\epsilon}_{n, t}\right)$ so

$$
\begin{gathered}
\left\|\sum_{t=1}^{k_{n}} a_{n, t} y_{t}\right\|_{1} \leq\left\|\sum_{t=1}^{k_{n}} a_{n, t}\left(\hat{\epsilon}_{n, t}-\mathbb{E}_{\mathcal{G}_{t-1}}\left(\hat{\epsilon}_{n, t}\right)\right)\right\|_{1}+\left\|\sum_{t=1}^{k_{n}} a_{n, t}\left(\tilde{\epsilon}_{n, t}-\mathbb{E}_{\mathcal{G}_{t-1}}\left(\tilde{\epsilon}_{n, t}\right)\right)\right\|_{1} \\
\leq\left\|\sum_{t=1}^{k_{n}} a_{n, t}\left(\hat{\epsilon}_{n, t}-\mathbb{E}_{\mathcal{G}_{t-1}}\left(\hat{\epsilon}_{n, t}\right)\right)\right\|_{2}+2\left(\sup _{n \in \mathbb{N}} \sum_{t=1}^{k_{n}}\left|a_{n, t}\right|\right) \sup _{t \in \mathbb{N}}\left\|\tilde{\epsilon}_{n, t}\right\|_{1}
\end{gathered}
$$

by the Lyapounov inequality and the Jensen inequality for conditional expectations. By $(11)$ and uniform integrability of $\left(\epsilon_{t}\right), \sup _{t \in \mathbb{N}}\left\|\tilde{\epsilon}_{n, t}\right\|_{1} \rightarrow 0$ so the second term on the right tends to 0 . For the first term, orthogonality of $\left\{\hat{\epsilon}_{n, t}-\mathbb{E}_{\mathcal{G}_{t-1}}\left(\hat{\epsilon}_{n, t}\right): t \geq 1\right\}$ yields

$$
\left\|\sum_{t=1}^{k_{n}} a_{n, t}\left(\epsilon_{1 t}-\mathbb{E}_{\mathcal{G}_{t-1}}\left(\epsilon_{1 t}\right)\right)\right\|_{2}^{2}=\sum_{t=1}^{k_{n}} a_{n, t}^{2} \mathbb{E}\left[\left(\epsilon_{1 t}-\mathbb{E}_{\mathcal{G}_{t-1}}\left(\epsilon_{1 t}\right)\right)^{2}\right] \leq \Delta_{n}^{2} \sum_{t=1}^{k_{n}} a_{n, t}^{2} \rightarrow 0
$$

by the choice of $\left(\Delta_{n}\right)_{n \in \mathbb{N}}$ in (11). This proves part (i). 
For part (ii), the fact that $\left(y_{t}\right)_{t \in \mathbb{Z}}$ is $\mathcal{G}_{t}$-adapted implies that, for any fixed integer $M>0$

$$
\sum_{t=1}^{k_{n}} a_{n, t} y_{t}=\sum_{m=0}^{M-1} \sum_{t=1}^{k_{n}} a_{n, t}\left(\mathbb{E}_{\mathcal{G}_{t-m}}\left(y_{t}\right)-\mathbb{E}_{\mathcal{G}_{t-m-1}}\left(y_{t}\right)\right)+\sum_{t=1}^{k_{n}} a_{n, t} \mathbb{E}_{\mathcal{G}_{t-M}}\left(y_{t}\right)
$$

as in equation (6) of Andrews (1988). For each $m$,

$$
\epsilon_{t}^{(m)}:=\mathbb{E}_{\mathcal{G}_{t-m}}\left(y_{t}\right)-\mathbb{E}_{\mathcal{G}_{t-m-1}}\left(y_{t}\right)
$$

is a $\mathcal{G}_{t-m}$-martingale difference process that inherits the uniform integrability property from $y_{t}$ and so $\epsilon_{t}^{(m)}$ satisfies the conclusion of part (i). Applying the triangle inequality and (7) to (12) we obtain

$$
\begin{aligned}
\left\|\sum_{t=1}^{k_{n}} a_{n, t} y_{t}\right\|_{1} & \leq \sum_{m=0}^{M-1}\left\|\sum_{t=1}^{k_{n}} a_{n, t} \epsilon_{t}^{(m)}\right\|_{1}+\sum_{t=1}^{k_{n}}\left|a_{n, t}\right|\left\|\mathbb{E}_{\mathcal{G}_{t-M}}\left(y_{t}\right)\right\|_{1} \\
& \leq M \max _{0 \leq m<M}\left\|\sum_{t=1}^{k_{n}} a_{n, t} \epsilon_{t}^{(m)}\right\|_{1}+\sup _{n \in \mathbb{N}} \sum_{t=1}^{k_{n}}\left|a_{n, t}\right| \sup _{t \in \mathbb{Z}} \zeta_{t} \psi_{M} \cdot(13)
\end{aligned}
$$

Let $\delta>0$ be arbitrary. Since $C:=\sup _{n \in \mathbb{N}} \sum_{t=1}^{k_{n}}\left|a_{n, t}\right| \sup _{t \in \mathbb{Z}} \zeta_{t}<\infty$ and $\psi_{M} \rightarrow 0$, there exists $M_{0}(\delta) \in \mathbb{N}$ such that $\psi_{M_{0}(\delta)} \leq \delta /(2 C)$. Choosing $M=M_{0}(\delta)$ in (13), we obtain

$$
\begin{aligned}
\left\|\sum_{t=1}^{k_{n}} a_{n, t} y_{t}\right\|_{1} & \leq M_{0}(\delta) \max _{0 \leq m<M_{0}(\delta)}\left\|\sum_{t=1}^{k_{n}} a_{n, t} \epsilon_{t}^{(m)}\right\|_{1}+\frac{\delta}{2} \\
& \leq M_{0}(\delta) \frac{\delta}{2 M_{0}(\delta)}+\frac{\delta}{2}=\delta
\end{aligned}
$$

where the second inequality applies for all but finitely many $n$ since $\max _{1 \leq m<M_{0}(\delta)}\left\|\sum_{t=1}^{k_{n}} a_{n, t} \epsilon_{t}^{(m)}\right\|_{1} \rightarrow 0$ by part (i).

\subsection{Proof of Lemma 2.}

Let us first establish some useful notation. As in $\mathrm{M}_{a}$, using the linear process representation of $u_{t}, Y_{n}(\kappa)$ and $Z_{n}(\kappa)$ of (8) are factored as the sum of 
pairs of uncorrelated components: $Z_{n}(\kappa)=Z_{n}^{(1)}(\kappa)+Z_{n}^{(2)}(\kappa)$ and $Y_{n}(\kappa)=$ $Y_{n}^{(1)}(\kappa)+Y_{n}^{(2)}(\kappa)$ with

$$
\begin{gathered}
Z_{n}^{(1)}(\kappa)=\frac{1}{n^{\left(\frac{3}{2}-\kappa\right) \alpha}} \sum_{t=1}^{\tau_{n}(\beta)} \rho_{n}^{-t} \sum_{j=0}^{t} c_{j} \varepsilon_{t-j}, \quad Z_{n}^{(2)}(\kappa)=\sum_{j=1}^{\infty} B_{n j}(\kappa) \varepsilon_{-j} \\
Y_{n}^{(1)}(\kappa)=\sum_{j=1}^{\tau_{n}(\beta)} C_{n j}(\kappa) \varepsilon_{n+1-j}, \quad Y_{n}^{(2)}(\kappa)=\sum_{k>\tau_{n}(\beta)} \sum_{t=1}^{\tau_{n}(\beta)} \frac{\rho_{n}^{-t} c_{k-t}}{n^{\left(\frac{3}{2}-\kappa\right) \alpha}} \varepsilon_{n+1-k}
\end{gathered}
$$

where $B_{n j}(\kappa)$ and $C_{n j}(\kappa)$ are arrays of real numbers defined by

$$
B_{n j}(\kappa)=n^{-\left(\frac{3}{2}-\kappa\right) \alpha} \sum_{t=1}^{\tau_{n}(\beta)} \rho_{n}^{-t} c_{t+j}, \quad C_{n j}(\kappa)=n^{-\left(\frac{3}{2}-\kappa\right) \alpha} \sum_{t=1}^{j} \rho_{n}^{-t} c_{j-t}
$$

and $\tau_{n}(\beta)$ is the sequence defined in $(9)$. Denote the array

$$
A_{n j}(\kappa)=n^{-\left(\frac{3}{2}-\kappa\right) \alpha} \rho_{n}^{-j} \sum_{i=0}^{\tau_{n}(\beta)} c_{i} \rho_{n}^{-i}
$$

related to $Z_{n}^{(1)}(\kappa)$. When $\kappa=1$, we write

$$
A_{n j}=A_{n j}(1), B_{n j}=B_{n j}(1) \text { and } C_{n j}=C_{n j}(1)
$$

As in $\mathrm{M}_{a}$, the asymptotic behaviour of $\left(Z_{n}, Y_{n}\right)$ is determined by $Z_{n}^{(1)}$ and $Y_{n}^{(1)}$ under Assumptions $\operatorname{LP}(\mathrm{i})$ and $\operatorname{LP}(\mathrm{iii})$, and by $Z_{n}^{(1)}, Z_{n}^{(2)}$ and $Y_{n}^{(1)}$ under Assumption LP(ii). $Y_{n}^{(2)}$ is asymptotically negligible in all cases. Finally, we say an array $\left(\psi_{n k}\right)$ of random vectors satisfies the Lindeberg condition if

$$
\sum_{k=0}^{n} \mathbb{E}_{\mathcal{F}_{k-1}}\left(\left\|\psi_{n k}\right\|^{2} \mathbf{1}\left\{\left\|\psi_{n k}\right\|>\delta\right\}\right) \rightarrow_{p} 0 \quad \forall \delta>0
$$

Proof of Lemma 2(i). Under Assumption $\mathrm{CH},\left(\varepsilon_{t}\right)_{t \in \mathbb{Z}}$ is a white noise process with variance equal to $\sigma^{2}$, so Lemmata B1 and B2 of $\mathrm{M}_{b}$ continue to 
apply. We have that

$$
\begin{aligned}
{\left[Y_{n}(1), Z_{n}(1)\right]^{\prime} } & =\left[Y_{n}^{(1)}(1), Z_{n}^{(1)}(1)\right]^{\prime}+o_{p}(1) \\
& =\left[\sum_{j=1}^{\tau_{n}(\beta)} C_{n j} \varepsilon_{n+1-j}, \sum_{j=0}^{\tau_{n}(\beta)} A_{n j} \varepsilon_{j}\right]^{\prime}+o_{p}(1) \\
& =\sum_{j=0}^{n} \zeta_{n j}+o_{p}(1)
\end{aligned}
$$

where $\zeta_{n j}$ denotes the $\mathcal{F}_{j}$-martingale difference array

$$
\zeta_{n j}=\left[\bar{C}_{n j}, \bar{A}_{n j}\right]^{\prime} \varepsilon_{j}
$$

with

$$
\bar{A}_{n j}=A_{n j} \mathbf{1}\left\{j \leq \tau_{n}(\beta)\right\} \quad \bar{C}_{n j}=C_{n, n+1-j} \mathbf{1}\left\{j>n-\tau_{n}(\beta)\right\}
$$

and $A_{n j}$ and $C_{n j}$ defined in (17), (16) and (18). By (9), $n-\tau_{n}(\beta)>\tau_{n}(\beta)$ so $\bar{U}_{n k} \bar{C}_{n k}=0$ for all $k$. By Assumption $\mathrm{CH}$ the conditional variance of $\sum_{k=0}^{n} \zeta_{n k}$ is given by

$$
U_{n}:=\sum_{j=0}^{n} \mathbb{E}_{\mathcal{F}_{j-1}} \zeta_{n j} \zeta_{n j}^{\prime}=\operatorname{diag}\left(\sum_{j=1}^{\tau_{n}(\beta)} C_{n j}^{2} h_{j}, \sum_{j=0}^{\tau_{n}(\beta)} A_{n j}^{2} h_{j}\right)
$$

By Lemma B2(ii) in $\mathrm{M}_{b}, U_{n} \rightarrow p \frac{\omega^{2}}{2 c} I_{2}$ provided that

$$
\left\|\sum_{j=1}^{\tau_{n}(\beta)} C_{n j}^{2}\left(h_{j}-\sigma^{2}\right)\right\|_{1}+\left\|\sum_{j=0}^{\tau_{n}(\beta)} A_{n j}^{2}\left(h_{j}-\sigma^{2}\right)\right\|_{1} \rightarrow 0 .
$$

To prove (21), we employ Lemma 1(ii) with the identifications $y_{t}:=h_{t}-\sigma^{2}$, $k_{n}:=\tau_{n}(\beta), \mathcal{G}_{t}:=\mathcal{F}_{t-1}$, and $a_{n, t} \in\left\{C_{n t}^{2}, A_{n t}^{2}\right\}$. Since $h_{t}$ is $\mathcal{F}_{t-1}$-adapted, (3) of Assumption $\mathrm{CH}$ implies that $y_{t}=h_{t}-\sigma^{2}$ satisfies (7) of Lemma 1. The sequences $\sum_{t=0}^{\tau_{n}(\beta)} A_{n t}^{2}$ and $\sum_{t=0}^{\tau_{n}(\beta)} C_{n t}^{2}$ are convergent by Lemma B2(ii) in $\mathrm{M}_{b}$ so the first condition of (6) is satisfied. For the second condition of (6), since $C:=\sum_{i=0}^{\infty}\left|c_{i}\right|<\infty$ we obtain

$$
\sum_{k=1}^{\tau_{n}(\beta)} C_{n k}^{4}=\frac{1}{n^{2 \alpha}} \sum_{k=1}^{\tau_{n}(\beta)}\left(\sum_{t=1}^{k} \rho_{n}^{-t} c_{k-t}\right)^{4} \leq C^{4} \frac{\tau_{n}(\beta)}{n^{2 \alpha}}=o\left(\frac{1}{n^{\alpha / 2}}\right)
$$


by (9) and, similarly,

$$
\sum_{k=1}^{\tau_{n}(\beta)} U_{n k}^{4}=\frac{1}{n^{2 \alpha}} \sum_{k=1}^{\tau_{n}(\beta)} \rho_{n}^{-4 k}\left(\sum_{j=0}^{\tau_{n}(\beta)} c_{j} \rho_{n}^{-j}\right)^{4} \leq \frac{C^{4}}{n^{2 \alpha}} \sum_{k=0}^{\infty} \rho_{n}^{-4 k}=O\left(\frac{1}{n^{\alpha}}\right) .
$$

This proves (21) and the required convergence $U_{n} \rightarrow_{p} \frac{\omega^{2}}{2 c} I_{2}$ for the conditional variance. By the proof of equation (11) in $\mathrm{M}_{b}$, it is clear that the array $\left(\zeta_{n j}\right)$ in (20) satisfies the Lindeberg condition (19) provided that $\left(\varepsilon_{t}^{2}\right)_{t \in \mathbb{Z}}$ is a uniformly integrable sequence. The latter is guaranteed by Assumption $\mathrm{CH}$ because of strict stationarity and integrability of $\left(\varepsilon_{t}^{2}\right)_{t \in \mathbb{Z}}$. The lemma now follows by applying the martingale CLT in Corollary 3.1 of Hall and Heyde (1980) to the martingale difference array $\left(\zeta_{n j}\right)$ in $(20)$.

Proof of Lemma 2(iii). From the proof of Lemma 4 of $\mathrm{M}_{b}$ (which only employs unconditional second moment bounds) we obtain

$$
\begin{aligned}
\frac{1}{\log n^{\alpha}}\left[Y_{n}(1), Z_{n}(1)\right] & =\frac{1}{\log n^{\alpha}}\left[Y_{n}^{(1)}(1), Z_{n}^{(1)}(1)\right]+o_{p}(1) \\
& =\frac{1}{\log n^{\alpha}} \sum_{j=0}^{n} \zeta_{n j}+o_{p}(1)
\end{aligned}
$$

with $\zeta_{n j}$ defined as in (20). By the argument of part (i), it is sufficient to verify (6) of Lemma 1 with the same identifications as in part (i), apart from $a_{n, t} \in\left\{\left(C_{n t} / \log n^{\alpha}\right)^{2},\left(A_{n t} / \log n^{\alpha}\right)^{2}\right\}$. The first part of (6) follows since $\left(\log n^{\alpha}\right)^{-2} \sum_{j=0}^{\tau_{n}(\beta)} C_{n t}^{2}$ and $\left(\log n^{\alpha}\right)^{-2} \sum_{j=0}^{\tau_{n}(\beta)} A_{n t}^{2}$ both converge to $\theta^{2} / 2 c$ (equations (16) and (17) of $\mathrm{M}_{b}$ ). For the second part of (6), since $\sum_{i=0}^{n}\left|c_{i}\right|=$ $O(\log n)$

$$
\begin{aligned}
\frac{1}{\left(\log n^{\alpha}\right)^{4}} \sum_{k=1}^{\tau_{n}(\beta)} C_{n k}^{4} & =\frac{1}{n^{2 \alpha}\left(\log n^{\alpha}\right)^{4}} \sum_{k=1}^{\tau_{n}(\beta)}\left(\sum_{t=1}^{k} \rho_{n}^{-t} c_{k-t}\right)^{4} \\
& \leq \frac{\left(\sum_{i=0}^{n}\left|c_{i}\right|\right)^{4}}{\left(\log n^{\alpha}\right)^{4}} \frac{\tau_{n}(\beta)}{n^{2 \alpha}}=o\left(\frac{1}{n^{\alpha / 2}}\right)
\end{aligned}
$$




$$
\begin{aligned}
\frac{1}{\left(\log n^{\alpha}\right)^{4}} \sum_{k=1}^{\tau_{n}(\beta)} A_{n k}^{4} & =\frac{1}{n^{2 \alpha}\left(\log n^{\alpha}\right)^{4}} \sum_{k=1}^{\tau_{n}(\beta)} \rho_{n}^{-4 k}\left(\sum_{j=0}^{\tau_{n}(\beta)} c_{j} \rho_{n}^{-j}\right)^{4} \\
& \leq \frac{\left(\sum_{i=0}^{n}\left|c_{i}\right|\right)^{4}}{\left(\log n^{\alpha}\right)^{4}} \frac{1}{n^{2 \alpha}} \sum_{k=0}^{\infty} \rho_{n}^{-4 k}=O\left(\frac{1}{n^{\alpha}}\right)
\end{aligned}
$$

Proof of Lemma 2(ii). First note that the definition of $A_{n j}(\kappa)$ in (17) differs from $A_{n j}$ in $\mathrm{M}_{a}$ by a slowly varying factor $L\left(n^{\alpha}\right)$. By Propositions 3.2.1-3.2.3 of $\mathrm{M}_{a}$,

$$
\begin{aligned}
L\left(n^{\alpha}\right)^{-1}\left[Z_{n}(\kappa), Y_{n}(\kappa)\right]^{\prime} & =L\left(n^{\alpha}\right)^{-1}\left[Z_{n}^{(1)}(\kappa), Z_{n}^{(2)}(\kappa), Y_{n}^{(1)}(\kappa)\right]+o_{p}(1) \\
& =\sum_{j=-\tau_{n}(\beta)}^{n} \xi_{n j}+o_{p}(1)
\end{aligned}
$$

where $\xi_{n k}:=\left[\tilde{A}_{n j}, \tilde{B}_{n j}, \tilde{C}_{n j}\right]^{\prime} \varepsilon_{j}$ is a $\mathcal{F}_{j}$-martingale difference array in $\mathbb{R}^{3}$ with components given by

$\tilde{A}_{n j}=L\left(n^{\alpha}\right)^{-1} A_{n j}(\kappa) \mathbf{1}\left\{0 \leq j \leq \tau_{n}(\beta)\right\}, \quad \tilde{B}_{n j}=L\left(n^{\alpha}\right)^{-1} B_{n,-j}(\kappa) \mathbf{1}\{j<0\}$,

$\tilde{C}_{n j}=L\left(n^{\alpha}\right)^{-1} C_{n, n+1-j}(\kappa) \mathbf{1}\left\{j>n-\tau_{n}(\beta)\right\}$

(see equation (23) of $\mathrm{M}_{a}$ ), with $A_{n j}(\kappa), B_{n j}(\kappa)$ and $C_{n j}(\kappa)$ defined in defined in (17) and (16). By (9), $n-\tau_{n}(\beta)>\tau_{n}(\beta)$ so $\tilde{A}_{n k} \tilde{B}_{n k}=\tilde{A}_{n k} \tilde{C}_{n k}=$ $\tilde{B}_{n k} \tilde{C}_{n k}=0$ for all $k$, so the conditional variance of the martingale array in (22) is given by

$$
\begin{aligned}
U_{n} & =\sum_{j=-\tau_{n}(\beta)}^{n} \mathbb{E}_{\mathcal{F}_{j-1}} \xi_{n j} \xi_{n j}^{\prime}=\sum_{j=-\tau_{n}(\beta)}^{n} \operatorname{diag}\left[\tilde{A}_{n j}^{2}, \tilde{B}_{n j}^{2}, \tilde{C}_{n j}^{2}\right] h_{j} \\
& =\frac{1}{L\left(n^{\alpha}\right)^{2}} \operatorname{diag}\left[\sum_{j=0}^{\tau_{n}(\beta)} A_{n j}^{2}(\kappa) h_{j}, \sum_{j=1}^{\tau_{n}(\beta)} B_{n j}^{2}(\kappa) h_{j}, \sum_{j=1}^{\tau_{n}(\beta)} C_{n j}^{2}(\kappa) h_{j}\right] .
\end{aligned}
$$

Denoting $y_{j}=h_{j}-\sigma^{2}$,

$$
\begin{aligned}
\tilde{U}_{n} & =\frac{1}{L\left(n^{\alpha}\right)^{2}} \operatorname{diag}\left[\sum_{j=0}^{\tau_{n}(\beta)} A_{n j}^{2}(\kappa) y_{j}, \sum_{j=1}^{\tau_{n}(\beta)} B_{n j}^{2}(\kappa) y_{j}, \sum_{k=1}^{\tau_{n}(\beta)} C_{n j}^{2}(\kappa) y_{j}\right] \\
U & =\frac{\sigma^{2} c^{2 \kappa-3} \Gamma(1-\kappa)^{2}}{2} \operatorname{diag}\left[1, \frac{1}{\cos \pi(1-\kappa)}-1, \frac{1}{\cos \pi(1-\kappa)}\right],
\end{aligned}
$$


Propositions 3.2.1-3.2.3 of $\mathrm{M}_{a}$, imply that

$$
U_{n}=U+\tilde{U}_{n}+o(1) .
$$

Therefore, $\tilde{U}_{n} \rightarrow{ }_{p} 0$ is sufficient for $U_{n} \rightarrow_{p} U$ as in Proposition 3.2.4 of $\mathrm{M}_{a}$. The proof of equation (24) of $\mathrm{M}_{a}$, shows that uniform integrability of $\left(\varepsilon_{t}^{2}\right)_{t \in \mathbb{Z}}$ (which is guaranteed by Assumption $\mathrm{CH}$ ) is sufficient for the array $\left(\xi_{n j}\right)$ in (22) to satisfy the Lindeberg condition (19). Thus, if $\tilde{U}_{n} \rightarrow{ }_{p} 0$ holds, the martingale CLT (Corollary 3.1 of Hall and Heyde (1980)) applied to (22) yields

$$
L\left(n^{\alpha}\right)^{-1}\left[Z_{n}^{(1)}(\kappa), Z_{n}^{(2)}(\kappa), Y_{n}^{(1)}(\kappa)\right] \Rightarrow\left[Z^{(1)}(\kappa), Z^{(2)}(\kappa), Y(\kappa)\right]
$$

for each $\kappa \in(1 / 2,1)$, as in Proposition 3.2.4 in $\mathrm{M}_{a}$ where $Z^{(1)}(\kappa), Z^{(2)}(\kappa)$ and $Y(\kappa)$ are independent zero mean Gaussian variates with variances $V_{\kappa}^{(1)}$, $V_{\kappa}-V_{\kappa}^{(1)}$ and $V_{\kappa}$ respectively, where $V_{\kappa}^{(1)}=\sigma^{2} c^{2 \kappa-3} \Gamma(1-\kappa)^{2} / 2$ and $V_{\kappa}$ is defined in (10). This completes the proof of the Lemma 2, provided that $\tilde{U}_{n} \rightarrow{ }_{p} 0$. To prove the latter, we employ Lemma 1(ii) to each term of (10), by taking $a_{n, j} \in\left\{\frac{A_{n j}^{2}(\kappa)}{L\left(n^{\alpha}\right)^{2}}, \frac{B_{n j}^{2}(\kappa)}{L\left(n^{\alpha}\right)^{2}}, \frac{C_{n j}^{2}(\kappa)}{L\left(n^{\alpha}\right)^{2}}\right\}$. The first part of (6) is satisfied since $\sum_{j=0}^{\tau_{n}(\beta)}\left|a_{n, j}\right|$ converges by Propositions 3.2.1-3.2.3 of $\mathrm{M}_{a}$. For the second part of (6), $\sum_{j=0}^{\tau_{n}(\beta)} a_{n, j}^{2}$ is bounded by:

$$
\begin{aligned}
\frac{1}{L\left(n^{\alpha}\right)^{4}} \sum_{j=0}^{\tau_{n}(\beta)} A_{n j}^{4}(\kappa) & =\frac{1}{L\left(n^{\alpha}\right)^{4} n^{(6-4 \kappa) \alpha}}\left(\sum_{i=0}^{\tau_{n}(\beta)} c_{i} \rho_{n}^{-i}\right)^{4} \sum_{j=0}^{\tau_{n}(\beta)} \rho_{n}^{-4 j} \\
& =\frac{1}{L\left(n^{\alpha}\right)^{4} n^{(6-4 \kappa) \alpha}} O\left(n^{4(1-\kappa) \alpha} L\left(n^{\alpha}\right)^{4}\right) \sum_{j=0}^{\tau_{n}(\beta)} \rho_{n}^{-4 j} \\
& =O(1) \frac{1}{n^{2 \alpha}} \sum_{j=0}^{\tau_{n}(\beta)} \rho_{n}^{-4 j}=O\left(\frac{1}{n^{\alpha}}\right)
\end{aligned}
$$


by Lemma A2(ii) of $\mathrm{M}_{a}$; since $t \mapsto L(t) t^{-\kappa}$ is non-increasing on $\left[t_{0},+\infty\right)$,

$$
\begin{aligned}
\frac{1}{L\left(n^{\alpha}\right)^{4}} \sum_{j=1}^{\tau_{n}(\beta)} B_{n j}^{4}(\kappa) & =\frac{1}{L\left(n^{\alpha}\right)^{4} n^{(6-4 \kappa) \alpha}} \sum_{j=1}^{\tau_{n}(\beta)}\left(\sum_{t=1}^{\tau_{n}(\beta)} \rho_{n}^{-t} c_{t+j}\right)^{4} \\
& =\frac{1}{L\left(n^{\alpha}\right)^{4} n^{(6-4 \kappa) \alpha}} \sum_{j=1}^{\tau_{n}(\beta)}\left[\left(\sum_{t=\left\lfloor t_{0}\right\rfloor+1}^{\tau_{n}(\beta)} \rho_{n}^{-t} L(t+j)(t+j)^{-\kappa}\right)+O(1)\right]^{4} \\
& \leq \frac{\tau_{n}(\beta)}{L\left(n^{\alpha}\right)^{4} n^{(6-4 \kappa) \alpha}}\left[\left(\sum_{t=\left\lfloor t_{0}\right\rfloor+1}^{\tau_{n}(\beta)} \rho_{n}^{-t} L(t) t^{-\kappa}\right)+O(1)\right]^{4} \\
& =\frac{\tau_{n}(\beta)}{L\left(n^{\alpha}\right)^{4} n^{(6-4 \kappa) \alpha}} O\left(n^{4(1-\kappa) \alpha} L\left(n^{\alpha}\right)^{4}\right) \\
& =O\left(\frac{\tau_{n}(\beta)}{n^{2 \alpha}}\right)=o\left(\frac{1}{n^{\alpha / 2}}\right)
\end{aligned}
$$

by Lemma A2(ii) of $\mathrm{M}_{a}$; by using the $C_{r}$-inequality with $r=4$ we obtain

$$
\begin{aligned}
\frac{1}{L\left(n^{\alpha}\right)^{4}} \sum_{j=1}^{\tau_{n}(\beta)} C_{n j}^{4}(\kappa) & =\frac{1}{L\left(n^{\alpha}\right)^{4} n^{(6-4 \kappa) \alpha}} \sum_{j=1}^{\tau_{n}(\beta)}\left(\sum_{t=1}^{j} \rho_{n}^{-t} c_{j-t}\right)^{4} \\
& =\frac{1}{L\left(n^{\alpha}\right)^{4} n^{(6-4 \kappa) \alpha}} \sum_{j=1}^{\tau_{n}(\beta)}\left(\sum_{t=0}^{j-1} \rho_{n}^{-(j-t)} c_{t}\right)^{4} \\
& \leq \frac{1}{L\left(n^{\alpha}\right)^{4} n^{(6-4 \kappa) \alpha}}\left(r_{1 n}+8 r_{2 n}+8 r_{3 n}\right)
\end{aligned}
$$

where

$$
\begin{aligned}
& r_{1 n}=\sum_{j=1}^{\left\lfloor n^{\alpha}\right\rfloor+1}\left(\sum_{t=0}^{j-1} \rho_{n}^{-(j-t)} c_{t}\right)^{4} \leq n^{\alpha}\left(\sum_{t=0}^{\left\lfloor n^{\alpha}\right\rfloor} c_{t}\right)^{4}=O\left(n^{\alpha} n^{4(1-\kappa) \alpha} L\left(n^{\alpha}\right)^{4}\right) \\
& r_{2 n}=\sum_{j=\left\lfloor n^{\alpha}\right\rfloor+2}^{\tau_{n}(\beta)}\left(\sum_{t=0}^{\left\lfloor n^{\alpha}\right\rfloor} \rho_{n}^{-(j-t)} c_{t}\right)^{4} \leq \tau_{n}(\beta)\left(\sum_{t=0}^{\left\lfloor n^{\alpha}\right\rfloor} c_{t}\right)^{4}=o\left(n^{3 \alpha / 2} n^{4(1-\kappa) \alpha} L\left(n^{\alpha}\right)^{4}\right)
\end{aligned}
$$

by (9) and

$$
r_{3 n}=\sum_{j=\left\lfloor n^{\alpha}\right\rfloor+2}^{\tau_{n}(\beta)}\left(\sum_{t=\left\lfloor n^{\alpha}\right\rfloor+1}^{j-1} \rho_{n}^{-(j-t)} c_{t}\right)^{4} .
$$


It clear that from the above bounds that the terms of (9) corresponding to $r_{1 n}$ and $r_{2 n}$ are of order $O\left(n^{-\alpha}\right)$ and $o\left(n^{-\alpha / 2}\right)$ respectively. It remains to estimate $r_{3 n}$. By Lemma A2(i) of $\mathrm{M}_{a}, \rho_{n}^{-(j-t)}=e^{-\frac{c}{n^{\alpha}}(j-t)}+O\left(n^{-\alpha / 2}\right)$ uniformly in $t$ and $j$

$$
\begin{aligned}
r_{3 n} & =\sum_{j=\left\lfloor n^{\alpha}\right\rfloor+2}^{\tau_{n}(\beta)}\left(\sum_{t=\left\lfloor n^{\alpha}\right\rfloor+1}^{j-1}\left[e^{-\frac{c}{n^{\alpha}}(j-t)}+O\left(n^{-\alpha / 2}\right)\right] c_{t}\right)^{4} \\
& \leq 8 \sum_{j=\left\lfloor n^{\alpha}\right\rfloor+2}^{\tau_{n}(\beta)}\left(\sum_{t=\left\lfloor n^{\alpha}\right\rfloor+1}^{j-1} e^{-\frac{c}{n^{\alpha}}(j-t)} c_{t}\right)^{4}+8 O\left(n^{-2 \alpha}\right) \sum_{j=\left\lfloor n^{\alpha}\right\rfloor+2}^{\tau_{n}(\beta)}\left(\sum_{t=\left\lfloor n^{\alpha}\right\rfloor+1}^{j-1} c_{t}\right)^{4} \\
& =8 r_{3 n}^{\prime}+O\left(n^{-2 \alpha}\right) O\left(n^{\beta} n^{4(1-\kappa) \beta} L\left(n^{\beta}\right)^{4}\right) \\
& =8 r_{3 n}^{\prime}+O\left(n^{(6-4 \kappa) \alpha}\right)
\end{aligned}
$$

because (9) implies that $4(1-\kappa)(\beta-\alpha) \leq 2(1-\kappa) \alpha$, so

$$
\begin{aligned}
n^{-(6-4 \kappa) \alpha} n^{-2 \alpha} n^{\beta} n^{4(1-\kappa) \beta} L\left(n^{\beta}\right)^{4} & =o\left(n^{-\alpha / 2}\right) n^{-2 \alpha} n^{4(1-\kappa)(\beta-\alpha)} L\left(n^{\beta}\right)^{4} \\
& \leq o\left(n^{-\alpha / 2} n^{-\kappa \alpha} L\left(n^{\beta}\right)^{4}\right) .
\end{aligned}
$$

To deal with the final remainder term $r_{3 n}^{\prime}$, we employ Euler summation:

$$
\begin{aligned}
& r_{3 n}^{\prime}=\sum_{j=\left\lfloor n^{\alpha}\right\rfloor+2}^{\tau_{n}(\beta)}\left(\sum_{t=\left\lfloor n^{\alpha}\right\rfloor+1}^{j-1} e^{-\frac{c}{n^{\alpha}}(j-t)} t^{-\kappa} L(t)\right)^{4} \\
& =\sum_{j=\left\lfloor n^{\alpha}\right\rfloor+2}^{\tau_{n}(\beta)}\left(\int_{\left\lfloor n^{\alpha}\right\rfloor+1}^{j-1} e^{-\frac{c}{n^{\alpha}}(j-\lfloor t\rfloor)}\lfloor t\rfloor^{-\kappa} L(\lfloor t\rfloor) d t\right)^{4} \\
& =n^{4 \alpha} \sum_{j=\left\lfloor n^{\alpha}\right\rfloor+2}^{\tau_{n}(\beta)}\left(\int_{\left(\left\lfloor n^{\alpha}\right\rfloor+1\right) / n^{\alpha}}^{(j-1) / n^{\alpha}} e^{-\frac{c}{n^{\alpha}}\left(j-\left\lfloor n^{\alpha} t\right\rfloor\right)}\left\lfloor n^{\alpha} t\right\rfloor^{-\kappa} L\left(\left\lfloor n^{\alpha} t\right\rfloor\right) d t\right)^{4} \\
& =n^{4 \alpha} \int_{\left\lfloor n^{\alpha}\right\rfloor+2}^{\tau_{n}(\beta)}\left(\int_{\left(\left\lfloor n^{\alpha}\right\rfloor+1\right) / n^{\alpha}}^{(\lfloor s\rfloor-1) / n^{\alpha}} e^{-\frac{c}{n^{\alpha}}\left(\lfloor s\rfloor-\left\lfloor n^{\alpha} t\right\rfloor\right)}\left\lfloor n^{\alpha} t\right\rfloor^{-\kappa} L\left(\left\lfloor n^{\alpha} t\right\rfloor\right) d t\right)^{4} d s \\
& =n^{5 \alpha} n^{-4 \kappa \alpha} L\left(n^{\alpha}\right)^{4} \int_{\left(\left\lfloor n^{\alpha}\right\rfloor+2\right) / n^{\alpha}}^{\tau_{n}(\beta) / n^{\alpha}}\left(\int_{\left(\left\lfloor n^{\alpha}\right\rfloor+1\right) / n^{\alpha}}^{\left(\left\lfloor n^{\alpha} s\right\rfloor-1\right) / n^{\alpha}} g_{n}(s, t) d t\right)^{4} d s \\
& \leq n^{\alpha} n^{4(1-\kappa) \alpha} L\left(n^{\alpha}\right)^{4} \int_{1}^{\infty}\left(\int_{1}^{s} g_{n}(s, t) d t\right)^{4} d s
\end{aligned}
$$


where, for all $t \geq 1$

$$
\begin{aligned}
g_{n}(s, t) & =e^{-\frac{c}{n^{\alpha}}\left(\left\lfloor n^{\alpha} s\right\rfloor-\left\lfloor n^{\alpha} t\right\rfloor\right)}\left(\frac{\left\lfloor n^{\alpha} t\right\rfloor}{n^{\alpha}}\right)^{-\kappa} \frac{L\left(\left\lfloor n^{\alpha} t\right\rfloor\right)}{L\left(n^{\alpha}\right)} \\
& =e^{-c\left(\frac{\left\lfloor n^{\alpha}\right\rfloor}{n^{\alpha} s}-\frac{\left\lfloor n^{\alpha} t\right\rfloor}{n^{\alpha} t}\right)} \frac{L\left(n^{\alpha}\right)}{L\left(\left\lfloor n^{\alpha}\right\rfloor\right)}\left(\frac{\left\lfloor n^{\alpha}\right\rfloor}{n^{\alpha}}\right)^{-\kappa} e^{-c(s-t)}\left(\frac{\left\lfloor n^{\alpha} t\right\rfloor}{\left\lfloor n^{\alpha}\right\rfloor}\right)^{-\kappa} \frac{L\left(\left\lfloor n^{\alpha} t\right\rfloor\right)}{L\left(\left\lfloor n^{\alpha}\right\rfloor\right)} \\
& \leq C_{1} e^{-c(s-t)}\left(\frac{\left\lfloor n^{\alpha} t\right\rfloor}{\left\lfloor n^{\alpha}\right\rfloor}\right)^{-\kappa} \frac{L\left(\left\lfloor n^{\alpha} t\right\rfloor\right)}{L\left(\left\lfloor n^{\alpha}\right\rfloor\right)} \\
& \leq C_{2} e^{-c(s-t)}\left(\frac{\left\lfloor n^{\alpha} t\right\rfloor}{\left\lfloor n^{\alpha}\right\rfloor}\right)^{-\kappa+\delta} \leq C e^{-c(s-t)} t^{-\kappa+\delta}
\end{aligned}
$$

eventually for arbitrary $\delta>0$ and $C_{1}, C_{2}, C$ uniform constants by Potter's Theorem (see BGT, Theorem 1.5.6.(i)). Using the above bound, the integral in (26) satisfies

$$
\int_{1}^{\infty}\left(\int_{1}^{s} g_{n}(s, t) d t\right)^{4} d s \leq C^{4} \int_{1}^{\infty} e^{-4 c s}\left(\int_{1}^{s} e^{c t} t^{-(\kappa-\delta)} d t\right)^{4} d s<\infty
$$

by choosing $\delta \in(0, \kappa-1 / 2)$, because $I=\int_{1}^{\infty} e^{-4 c s}\left(\int_{1}^{s} e^{c t} t^{-\lambda} d t\right)^{4} d s<\infty$ for any $\lambda>1 / 2$. To see this, note that $\lim _{s \rightarrow \infty} e^{-c s} \int_{1}^{s} e^{c t} t^{-\lambda} d t=0$ by L'Hospital's rule; applying integration by parts twice we obtain

$$
\begin{aligned}
I & =\frac{1}{c} \int_{1}^{\infty} e^{-3 c s} s^{-\lambda}\left(\int_{1}^{s} e^{c t} t^{-\lambda} d t\right)^{3} d s \\
& =\frac{1}{3 c^{2}} \int_{1}^{\infty} e^{-3 c s}\left\{3 s^{-2 \lambda} e^{c t}\left(\int_{1}^{s} e^{c t} t^{-\lambda} d t\right)^{2}-\lambda s^{-\lambda-1}\left(\int_{1}^{s} e^{c t} t^{-\lambda} d t\right)^{3}\right\} d s \\
& =\frac{1}{c^{2}} \int_{1}^{\infty} s^{-2 \lambda}\left(\int_{1}^{s} e^{-c(s-t)} t^{-\lambda} d t\right)^{2} d s-\frac{\lambda}{3 c^{2}} \int_{1}^{\infty} s^{-\lambda-1}\left(\int_{1}^{s} e^{-c(s-t)} t^{-\lambda} d t\right)^{3} d s \\
& \leq \frac{1}{c^{2}} \int_{1}^{\infty} s^{-2 \lambda} d s\left(\int_{0}^{\infty} e^{-c u} d u\right)^{2}<\infty .
\end{aligned}
$$

By (26) we conclude that $r_{3 n}^{\prime}=O\left(n^{\alpha} n^{4(1-\kappa) \alpha} L\left(n^{\alpha}\right)^{4}\right)$ and standardising by the normalisation of $(25), L\left(n^{\alpha}\right)^{-4} n^{-(6-4 \kappa) \alpha} r_{3 n}^{\prime}=O\left(n^{-\alpha}\right)$. This shows that the right side of (25) tends to 0 as $n \rightarrow \infty$ and completes the proof of the lemma. 
Proof of Lemma 3. Having established the joint asymptotic behaviour of $L\left(n^{\alpha}\right)^{-1} Y_{n}(\kappa)$ and $L\left(n^{\alpha}\right)^{-1} Z_{n}(\kappa)$ under Assumption CH in Lemma 2, the proof of Lemma 3 follows the same steps as the proof of Lemma 5 of $\mathrm{M}_{a}$ : see page 185 of $\mathrm{M}_{a}$.

\section{References.}

Aue, A. and L. Horváth (2007). A limit theorem for mildly explosive autoregression with stable errors. Econometric Theory 23, 201-220.

Anderson, T.W. (1959). On asymptotic distributions of estimates of parameters of stochastic difference equations. Annals of Mathematical Statistics, 30, 676-687.

Andrews, D. W. (1988). Laws of large numbers for dependent non-identically distributed random variables. Econometric Theory, 4(3), 458-467.

Bingham, N.H., Goldie, C.M. and J.L. Teugels (1987). Regular Variation. CUP.

Bollerslev, T., Sizova, N., \& Tauchen, G. (2011). Volatility in equilibrium: Asymmetries and dynamic dependencies. Review of Finance, 16(1), 31-80.

Carrasco, M., \& Chen, X. (2002). Mixing and moment properties of various GARCH and stochastic volatility models. Econometric Theory, 18(1), 17-39.

Davidson, J. (1994). Stochastic limit theory: An introduction for econometricians. OUP Oxford.

Ghysels, E., Harvey, A. C., \& Renault, E. (1996). Stochastic volatility. Handbook of statistics, 14, 119-191.

Giraitis, L., Koul, H.L., D. Surgailis (1996). Asymptotic normality of regression estimators with long memory errors. Statistics and Prob. Letters, $29,317-335$.

Giraitis, L., Kokoszka, P., \& Leipus, R. (2000). Stationary ARCH models: dependence structure and central limit theorem. Econometric Theory, $16(1), 3-22$. 
Giraitis, L. and P. C. B. Phillips (2006). Uniform Limit Theory for Stationary Autoregression. Journal of Time Series Analysis, 27, 51-60.

Goldie, C. M. (1991). Implicit renewal theory and tails of solutions of random equations. The Annals of Applied Probability, 126-166.

Hall, P. and C.C. Heyde (1980). Martingale Limit Theory and its Application. AP.

Ibragimov, I. A. and Linnik, I. U. V. (1971). Independent and stationary sequences of random variables. Groningen: Wolters-Noordhoff.

Lee, J. H. (2017). Limit theory for explosive autoregression under conditional heteroskedasticity. Journal of Statistical Planning and Inference, Forthcoming.

Magdalinos, T. (2009). Appendix to "Mildly explosive autoregression under weak and strong dependence".

Magdalinos, T. and P. C. B. Phillips (2008). Limit Theory for Cointegrated Systems with Moderately Integrated and Moderately Explosive Regressors. Econometric Theory, 25, 482-526.

Magdalinos, T. (2012). Mildly explosive autoregression under weak and strong dependence. Journal of Econometrics, 169(2), 179-187.

McLeish, D. L. (1975). A maximal inequality and dependent strong laws. The Annals of Probability, 3(5), 829-839.

Oh, H., Lee, S., \& Chan, N. H. (2017). Mildly explosive autoregression with mixing innovations. Journal of the Korean Statistical Society, Forthcoming.

Phillips, P. C. B. and T. Magdalinos (2007a). Limit theory for Moderate deviations from a unit root. Journal of Econometrics, 136, 115-130.

Phillips, P. C. B. and T. Magdalinos (2007b). Limit theory for Moderate deviations from a unit root under weak dependence. G. D. A. Phillips and E. Tzavalis (Eds.) The Refinement of Econometric Estimation and Test Procedures. CUP. 
Phillips, P.C.B., Wu, Y. and J. Yu (2009). Explosive behavior in the 1990s NASDAC: When did exuberance escalate asset values? Cowles discussion paper 1699 .

Phillips, P. C., \& Yu, J. (2011). Dating the timeline of financial bubbles during the subprime crisis. Quantitative Economics, 2(3), 455-491.

Samorodnitsky, G. (2006). Long Range Dependence. Now Publishers.

Straumann, D. (2004). Estimation in Conditionally Heteroscedastic Time Series Models. Springer.

White, J. S. (1958). The limiting distribution of the serial correlation coefficient in the explosive case. Annals of Mathematical Statistics 29, $1188-1197$.

Wu, W.B. and W. Min (2005). On linear processes with dependent innovations. Stochastic Processes and their Applications 115, 939-958. 